\title{
Influencia de parámetros clínicos sobre la severidad de la recesión gingival localizada
}

\section{Clinical parameters related to severity of localised gingival recession}

\author{
GARCÍA-RUBIO A* \\ BUJALDÓN-DAZA AL** \\ RODRÍGUEZ-ARCHILLA $\mathbf{A} * * *$
}

\begin{abstract}
García-Rubio A, Bujaldón-Daza AL, Rodríguez-Archilla A. Influencia de parámetros clínicos sobre la severidad de la recesión gingival localizada. Av Periodon Implantol. 2015; 27, 2: 67-73.
\end{abstract}

\begin{abstract}
RESUMMN
Introducción: La recesión gingival localizada es un problema de salud bucodental fundamental porque su progresión conduce a la pérdida dentaria. El propósito de este trabajo fue evaluar la influencia de distintos parámetros clínicos sobre la recesión gingival localizada.

Material y métodos: Se estudiaron 40 pacientes con recesión gingival localizada en los que se analizaron diversos parámetros clínicos relacionados con la recesión gingival. Para la comparación de variables cuantitativas se utilizó el test de Student (t-Student) y para la de variables cualitativas el test chi-cuadrado.

Resultados: En este estudio, según la clasificación de Miller, el 37,5\% de los pacientes tenían recesiones de tipo I, el 60\% de tipo II y el 2,5\% restante, de tipo III. Los siguientes parámetros: edad, frecuencia de cepillado, tipo de cepillo, uso de colutorios y de seda dental, otros hábitos orales y el tratamiento de ortodoncia, no tienen influencia sobre la severidad de la recesión gingival ( $p>0,05$ en todos los casos). Presentan recesión gingival más severa, los varones $(p=0,03)$, los sujetos fumadores $(p=0,007)$ y los que tienen técnicas de cepillado horizontal $(p=0,02)$. En los cuatro momentos de seguimiento del estudio (inicial, 6, 12 y 18 meses), la profundidad de sondaje, la pérdida de inserción, el índice de placa y el índice de sangrado mejoraron a los 6 meses para luego empeorar en el resto de períodos.

Discusión: El sexo, el tabaco y la técnica de cepillado son los tres factores que tienen influencia sobre la severidad de la recesión gingival localizada en este estudio.
\end{abstract}

PALABRAS CLAVE: Índice de placa, índice de sangrado, profundidad de sondaje, recesión gingival.

\section{SUMMMARY}

Introduction: Localised gingival recession is a major problem of oral health because its progression leads to the dental loss. The aim of this study was to evaluate the possible influence of different clinical parameters in localised gingival recession.

Material and methods: Forty patients with localised gingival recession were studied. Data from several clinical parameters related to gingival recession were collected.

Results: In this study, $37.5 \%$ of patients had recessions of Miller's type I, $60 \%$ Miller's type II, and the remaining $2.5 \%$ of Miller's type III. Age, frequency of toothbrushing, toothbrushing technique, use of mouthwash and/or dental floss, other oral habits, and orthodontic therapy, have no influence on the severity of gingival recession ( $p>0.05$ in all cases). More severe gingival recession was observed in males $(p=0.03)$, smokers $(p=0.007)$ and those with horizontal toothbrushing techniques $(p=0.02)$. In the four follow-up periods of the study, probing depth, attachment loss, plaque index and bleeding index improved after 6 months and then got worse in the rest of the follow-up intervals. 
Discussion: Gender, smoking and toothbrushing technique were the three factors that influenced the severity of localised gingival recession.

KEY WORDS: Dental plaque index, gingival recession, periodontal index, periodontal pocket.

Fecha de recepción: 1 de enero de 2014.

Fecha de aceptación: 21 de enero de 2014.

\section{INTRODUCCIÓN}

La recesión gingival es el desplazamiento del margen gingival apicalmente desde la unión cementoadamantina o desde la localización anterior de este límite en la cual las restauraciones han distorsionado la forma o apariencia de esta unión. La recesión gingival puede ser localizada o generalizada y estar asociada con una o más superficies (1). La exposición radicular resultante no es estéticamente agradable y podría conducir a sensibilidad y caries radicular.

Desde el punto de vista clínico, la clasificación de Miller (2) es probablemente la más usada de forma común para clasificar las recesiones gingivales. Esta clasificación, de acuerdo con la extensión de la recesión, contempla cuatro clases: I, II, III y IV.

La aparición y severidad de la recesión gingival puede verse condicionada por diversos factores. Entre estos destacan: la edad, donde diversos estudios (3-5) ponen de manifiesto que conforme aumenta la edad, aumenta la aparición y la severidad de la recesión gingival; el sexo $(3,4)$ estableciendo que la recesión gingival es mayor en los hombres que en las mujeres de la misma edad. Otros factores generales relacionados son: las enfermedades generales y el consumo de fármacos; los hábitos nocivos (consumo de tabaco y/o alcohol).

Los hábitos de higiene oral pueden jugar un papel importante en la etiología de la recesión, sobre todo al considerar la frecuencia y la técnica de cepillado, el tipo de cepillo y el uso de colutorios y/o seda dental. Se ha contrastado que la recesión gingival en personas con buena higiene oral afecta más a las superficies bucales que a las proximales o linguales $(6,7)$ y además es más frecuente $(4,8)$. Algunos estudios ( 9 ) establecen una correlación directa entre la recesión gingival y la frecuencia del cepillado dental.

También se han considerado que son factores asociados con la recesión gingival el uso de palillo de dientes o de piercings orales (10).
Los tratamientos de ortodoncia han sido citados como una causa de recesión localizada, pero no existen evidencias claras que atestigüen esta relación. Unos estudios no encontraron ninguna relación directa $(11,12)$, otros sí $(13,14)$.

La principal opción terapéutica para las recesiones es el injerto de tejido conectivo subepitelial que consigue una cobertura radicular predecible con un alto nivel cosmético $(15,16)$. Existen otras técnicas como los injertos de encía libre, los colgajos pediculados o la regeneración tisular guiada (17). El objetivo de este estudio fue evaluar la influencia de distintos parámetros clínicos sobre la recesión gingival localizada.

\section{MATERIAL Y MÉTODOS}

La muestra de este estudio estaba compuesta por 40 pacientes con recesión gingival localizada que acudieron para recibir tratamiento periodontal a un centro odontológico privado. De ellos, 30 (el 75\%) eran mujeres y 10 (un 25\%) varones, con edades comprendidas entre los 16 y los 73 años y una media de edad de $39,08 \pm 12,40$ años. Fueron excluidos del mismo los pacientes con recesiones generalizadas consecuencia de antecedentes periodontales y los portadores de piercings orales. Todos fueron explorados y diagnosticados por el mismo operador (AGR). Además, a cada uno de ellos se le proporcionó la información necesaria acerca de los propósitos de este trabajo con el fin de obtener su consentimiento informado.

Todos los pacientes de este estudio fueron evaluados en cuatro periodos diferentes: momento inicial del estudio, a los 6 , a los 12 y a los 18 meses.

En la primera visita (momento inicial), a cada paciente se le aplicó un protocolo clínico, que incluía datos de su historia clínica (patología sistémica previa, tratamientos médicos, consumo de tabaco y/o alcohol, frecuencia de cepillado, técnica de cepillado, tipo de cepillo, uso de colutorios y de seda dental, otros hábi- 
tos orales y tratamientos odontológicos previos con especial referencia a la ortodoncia).

Asimismo se le realizó un registro de los índices de placa y de sangrado, la medición de la profundidad de sondaje y de la pérdida de inserción. También se recogió el número de dientes con recesión localizada la clasificación de la misma de acuerdo con la clasificación de Miller y la cantidad de encía insertada.

Todos los pacientes fueron instruidos en técnicas de higiene bucodental adaptadas a sus condiciones y factores de riesgo. Se les instruyó para realizar cepillado 3 veces al día, utilizando técnicas de cepillado interproximal al menos una vez al día. En algunos casos, los pacientes fueron adiestrados en el uso de cepillo eléctrico para evitar fuerzas de presión excesivas.

En este trabajo, se realizó una estadística descriptiva (media aritmética, desviación estándar y porcentajes) y una estadística analítica (t-Student, test ANOVA, test de Wilcoxon y test chi-cuadrado). Se consideró como nivel mínimo de significación un valor de $\mathrm{p}<0,05$.

\section{RESULTADOS}

Los datos más relevantes de parámetros clínicos analizados se muestran en la tabla 1.

Los pacientes de este trabajo tienen un número medio de dientes con recesión localizada de 3,30 dientes, con un rango que oscila entre 1 y 8 dientes. Ninguno de los

\section{TABLA 1.- PRINCIPALES PARÁMETROS CLÍNICOS ANALIZADOS}

\begin{tabular}{|l|c|c|}
\hline Parámetro & Respuesta & Porcentaje \\
\hline Enfermedades generales & No & $82,5 \%$ \\
\hline Hábito de fumar & No & $62,5 \%$ \\
\hline Frecuencia de cepillado & $\begin{array}{c}\text { 3 o más } \\
\text { veces/día }\end{array}$ & $60,0 \%$ \\
\hline Técnica de cepillado & Vertical & $50,0 \%$ \\
\hline Tipo de cepillo dental & $\begin{array}{c}\text { Dureza } \\
\text { media }\end{array}$ & $47,5 \%$ \\
\hline Uso de seda dental & Sí & $52,5 \%$ \\
\hline Uso de colutorios & No & $72,5 \%$ \\
\hline
\end{tabular}

parámetros analizados en este estudio influye sobre el número medio de dientes con recesión gingival ( $p>0,05$ en todos los casos). En el caso de los tratamientos de ortodoncia tampoco se encontró relación estadísticamente significativa $(p=0,37)$ contradiciendo, en principio, la consideración de la ortodoncia como un factor etiológico fundamental en la aparición de la recesión gingival localizada.

En cuanto a la severidad de la recesión gingival de todos los sujetos participantes en el estudio, 15 (37,5\%) tenían recesiones de tipo I, $24(60 \%)$ de tipo II y 1 solo paciente $(2,5 \%)$ de tipo III. Ningún paciente presentó recesión tipo IV de Miller. Los siguientes parámetros: edad, frecuencia de cepillado, tipo de cepillo, uso de colutorios y de seda dental, otros hábitos orales y ortodoncia, no tienen influencia sobre la severidad de la recesión gingival ( $p>0,05$ en todos los casos).

Cuando se analiza la posible influencia del sexo sobre la severidad de la recesión gingival, se comprueba como casi la mitad de las mujeres presentan recesiones tipo I; mientras que las tipo II afecta al $80 \%$ de los varones, existiendo diferencias significativas $(p=0,03)$. Los hombres presentan una recesión gingival más severa.

Al relacionar la severidad de la recesión con el consumo de tabaco, se observa que la recesión empeora conforme aumenta el consumo y la antigüedad en el hábito tabáquico $(p=0,007)$, los fumadores tienen recesiones gingivales más severas.

Otro de los factores que influyen en la severidad de la recesión es la técnica de cepillado, observándose las recesiones más graves en los pacientes con técnicas de cepillado horizontal $(p=0,02)$.

Los datos correspondientes a la evolución de la profundidad de sondaje en los distintos momentos del estudio se muestran en la tabla 2.

Como se puede apreciar en la tabla 2 en cuanto a la evolución de la profundidad al sondaje en el momento inicial, a los 6, 12 y 18 meses, en las bolsas de 1 a 3 $\mathrm{mm}$ y en las de 4 a $6 \mathrm{~mm}$ se observan variaciones estadísticamente significativas $(p<0,001)$. Esto no ocurre con las bolsas de más de $6 \mathrm{~mm}(\mathrm{p}=0,93)$.

El mayor número de sujetos con bolsas mayores de 6 $\mathrm{mm}$ se observó entre aquellos que utilizaban cepillos de dureza media $(p=0,005)$. Cuando se relaciona la profundidad de sondaje con el tratamiento ortodóntico se aprecian diferencias significativas en los pacien- 


\begin{tabular}{|l|c|c|c|}
\hline \multicolumn{4}{|c|}{ TABLA 2.- EVOLUCIÓN DE LA } \\
PROFUNDIDAD DE SONDAJE SEGÚN \\
LOS MOIMENTOS DEL ESTUDIO
\end{tabular}

tes con bolsas tanto de 1 a $3 \mathrm{~mm}(\mathrm{p}=0,02)$, como de $4 \mathrm{a}$ $6 \mathrm{~mm}$ de profundidad ( $\mathrm{p}=0,01)$. De igual forma, la mayoría de los pacientes con bolsas mayores de $6 \mathrm{~mm}$ tienen recesión gingival más grave (tipo III de Miller) existiendo diferencias significativas $(p=0,003)$.

Los datos de la evolución de la pérdida de inserción se exponen en la tabla 3.

Respecto a la pérdida de inserción en el momento inicial, a los 6, 12 y 18 meses, no se aprecian variaciones estadísticamente significativas en ningún caso $(p=0,33$; 0,17 y 0,35 respectivamente). No obstante se constata una disminución progresiva en las pérdidas de inserción superiores a $6 \mathrm{~mm}$ en los 4 momentos del estudio, tal y como se muestra en la tabla 3.

Al asociar la pérdida de inserción con la dureza del cepillo, se encontró que en el caso de las pérdidas de inserción de más de $6 \mathrm{~mm}$ existe asociación significa-

\section{TABLA 3.- EVOLUCIÓN DE LA PÉRDIDA DE INSERCIÓN ATENDIENDO AL MOIMENTO DEL ESTUDIO}

\begin{tabular}{|l|ccc|}
\hline \multirow{2}{*}{ Momento } & \multicolumn{3}{|c|}{ Pérdida de inserción } \\
\cline { 2 - 5 } & $\mathbf{1 - 3} \mathbf{~ m m}$ & $\mathbf{4 - 6} \mathbf{~ m m}$ & $>\mathbf{6 ~ m m}$ \\
\hline Inicio & $94,68 \pm 5,25 \%$ & $4,95 \pm 4,39 \%$ & $0,45 \pm 1,46 \%$ \\
6 meses & $93,70 \pm 4,98 \%$ & $6,10 \pm 4,74 \%$ & $0,20 \pm 0,60 \%$ \\
12 meses & $93,83 \pm 4,96 \%$ & $6,03 \pm 4,76 \%$ & $0,15 \pm 0,70 \%$ \\
18 meses & $93,88 \pm 5,09 \%$ & $6,03 \pm 4,97 \%$ & $0,10 \pm 0,44 \%$ \\
Significación & $\mathrm{p}=0,33$ & $\mathrm{p}=0,17$ & $\mathrm{p}=0,35$ \\
\hline
\end{tabular}

tiva entre ambas variables $(p=0,01)$, aumentando la pérdida de inserción conforme aumenta la dureza del cepillo.

Al comparar las pérdidas de inserción con la presencia de tratamiento de ortodoncia se halló relación estadísticamente significativa $(p<0,05)$. Al igual que sucede con la profundidad de sondaje, los pacientes que presentaban tratamiento de ortodoncia también tenían un porcentaje mayor de pérdidas; esto puede ser debido a que este tipo de pacientes presentan una mayor retención de placa junto con peores condiciones para la higiene oral, lo que conlleva un aumento en la pérdida de inserción.

El número medio de dientes con ausencia de encía insertada que presenta cada paciente es de 0,85 dientes con un mínimo de 0 y un máximo de 8 dientes.

Los datos correspondientes a la evolución de los índices de placa y de sangrado en los distintos períodos de seguimiento del estudio se representan en la tabla 4.

Tal y como aparece recogido en la tabla 4 en los cuatro momentos evaluados (inicial, 6 meses, 12 meses y 18 meses), el índice de placa descendió a los 6 meses para subir a su nivel máximo a los 12 meses y luego descender levemente, existiendo diferencias altamente significativas $(p<0,001)$. Con respecto al índice de sangrado ocurre algo similar al índice de placa, desciende a los 6 meses para subir a su máximo nivel a los 12 meses y luego descender levemente, hallándose una relación muy significativa en este caso $(p=0,002)$.

Cuando se comparó el índice de placa a los 6 meses con la gravedad de la recesión, se encontró asocia-

\begin{tabular}{|c|c|c|}
\hline \multicolumn{3}{|c|}{$\begin{array}{c}\text { TABLA 4.- EVOLUCIÓN DE LOS } \\
\text { ÍNDICES DE PLACA Y DE SANGRADO } \\
\text { EN LOS DIFERENTES IMOIMENTOS } \\
\text { DEL ESTUDIO }\end{array}$} \\
\hline Momento & Índice de placa & Índice de sangrado \\
\hline Inicio & $27,18 \pm 4,86 \%$ & $11,15 \pm 3,89 \%$ \\
\hline 6 meses & $25,28 \pm 3,78 \%$ & $10,45 \pm 3$ \\
\hline 12 meses & $29,38 \pm 4,82 \%$ & $12,63 \pm 4,27 \%$ \\
\hline 18 meses & $28,85 \pm 4,57 \%$ & $12,30 \pm 3,91 \%$ \\
\hline Significación & $\mathrm{p}<0,001$ & $\mathrm{p}=0,002$ \\
\hline
\end{tabular}


ción significativa entre ambas variables $(p=0,01)$. Se puede apreciar que, a medida que aumenta la gravedad en la recesión, se incrementan los porcentajes del índice de placa.

Al relacionar el índice de sangrado a los 18 meses con la técnica de cepillado se encontraron diferencias estadísticamente significativas entre ambos parámetros $(p=0,04)$. Los pacientes que no se cepillaban presentaban un mayor porcentaje de sangrado y sorprendentemente, la técnica de cepillado horizontal fue la que produjo un menor sangrado.

\section{DISCUSIÓN}

La recesión gingival es un efecto indeseable de la exposición de la superficie radicular. Tiene un efecto antiestético y puede conducir al desarrollo de sensibilidad dental, junto con una mayor predisposición a la abrasión y a la caries radicular (18). Es un problema oral muy frecuente que afecta al $88 \%$ de los sujetos mayores de 65 años y casi a la mitad de la población entre los 18 y los 64 años (4). La presencia y extensión de la recesión gingival aumenta con la edad. Entre los factores clásicamente relacionados con la recesión gingival se encuentran: el trauma, el sexo, los dientes malposicionados, la inflamación y el consumo de tabaco. Esta recesión aparece en sujetos tanto con buena como con deficiente higiene oral. Se piensa que la recesión gingival tiene una etiología multifactorial en la que se ven implicados factores anatómicos, fisiológicos y patológicos. La recesión es más frecuente en las superficies bucales o vestibulares de los dientes (19).

Se han propuesto muchos factores como influyentes del desarrollo de la recesión del tejido marginal y existe controversia sobre el concepto de una adecuada zona de encía insertada (20). Actualmente las dimensiones de las diferentes partes de la mucosa masticatoria se han convertido en un tema de considerable interés en periodoncia desde un punto de vista epidemiológico y terapéutico. El grosor de la mucosa masticatoria tiene influencia sobre el desarrollo de recesiones gingivales y facilita la realización de tratamientos de cobertura radicular con injertos, etc. (21).

Como se ha dicho anteriormente, el desarrollo de la recesión gingival está condicionado por factores anatómicos (estructura del hueso alveolar, posición y forma del diente, patrón de erupción...), fisiológicos (movimiento dentario inducido) y patológicos (enfer- medades sistémicas previas, enfermedad periodontal inducida microbiológicamente y hábitos nocivos). El trauma y la abrasión de los tejidos duros y blandos orales producida por el cepillado parecen estar directamente relacionadas con el desarrollo de recesión gingival.

Aunque la abrasión dentaria es una parte integrante de la etiología de la recesión gingival, ésta no siempre es inducida por el cepillado. De hecho, según la literatura, esta relación cepillado-recesión gingival no es tan evidente. Si este cepillado es muy intenso puede exponer la unión amelocementaria produciendo una destrucción del periodonto de soporte que conduce a la recesión (22).

En el estudio de Susin y cols. (23), la prevalencia, extensión y severidad de la recesión gingival está correlacionada con la edad y con el sexo. Una mayor edad y el sexo masculino son dos de los factores relacionados con la aparición de recesión gingival. De igual forma se observa un mayor número de dientes con recesión gingival en los individuos de un nivel socioeconómico bajo, un deficiente cuidado bucodental y con hábitos nocivos como el consumo tabáquico.

La presencia de cálculo supragingival es otro de los factores más influyentes sobre la recesión. Los altos niveles de cálculo supragingival y el hábito de fumar son dos factores significativamente asociados a la aparición de recesión gingival.

Lang y Löe (24) estudiaron la cantidad mínima que se necesitaba de encía queratinizada compatible con la salud gingival. Los autores sugirieron que se necesitaba un mínimo de $2 \mathrm{~mm}$ para mantener una adecuada salud. Pero estudios longitudinales más recientes $(11,25)$ demostraron que la recesión de tejido blando no es mayor en las superficies vestibulares de dientes con una mínima cantidad de encía que en los que tienen una banda ancha. Lo que sí parece darse es mayor frecuencia de síntomas inflamatorios como enrojecimiento e inflamación. Dichos resultados también son similares en estudios realizados con implantes (26). En diversos estudios con implantes se ha demostrado que la colocación inmediata está asociada con una mayor frecuencia de recesión gingival en comparación con los que se colocan tras un periodo postextracción (27). No existe una regla básica para decidir qué cantidad de encía insertada se necesita sino que el factor determinante será la capacidad de controlar la placa bacteriana en dichas localizaciones por el paciente. 
El tabaco puede influir negativamente en la reducción de la recesión gingival y en el nivel de ganancia de inserción clínica. Adicionalmente, es más probable que los fumadores alcancen un menor porcentaje de localizaciones con cobertura radicular completa (28). El éxito del recubrimiento radicular estará determinado por el grado de afectación del hueso interproximal y es factible conocer de antemano las posibilidades de recubrimiento dependiendo de la clasificación de la recesión.

En conclusión, en este estudio, los dientes que presentaron más recesión fueron el incisivo central inferior izquierdo, el primer premolar inferior izquierdo y los incisivos central y lateral inferiores derechos. La clase II de Miller fue el tipo de recesión gingival más prevalente.

La edad, el sexo, el consumo de tabaco, la frecuencia y la técnica de cepillado, el tipo de cepillo, el uso de colutorios y/o de seda dental, otros hábitos orales y el tratamiento ortodóncico previo, no tienen influencia sobre la recesión gingival.

Tanto la profundidad de las bolsas como la pérdida de inserción están condicionadas, en el momento inicial, por la dureza del cepillo $(\mathrm{p}<0,05)$ y, en todos los períodos evaluados, por el tratamiento de ortodoncia $(p<0,05)$.

\section{BIBLIOGRAFÍA}

1. Smith RG. Gingival recession: reappraisal of an enigmatic condition and a new index for monitoring. J Clin Periodontol 1997;24:201-5.

2. Miller PD. A classification of marginal tissue recession. Int J Periodontics Restorative Dent 1985;5:9-13.

3. Albandar JM, Kingman A. Gingival recession, gingival bleeding, and dental calculus in adults 30 years of age and older in the United States 1988-1994. J Periodontol 1999;70:30-43.

4. Gorman WJ. Prevalence and etiology of gingival recession. J Periodontol 1967;38:316-22.

5. Murray JJ. Gingival recession in tooth types in high fluoride and low fluoride areas. J Periodontal Res 1973;8:243-51.

6. Löe H, Anerud A, Boysem H, Smith M. The natural history of periodontal disease in man: the rate of periodontal destruction before 40 years of age. J Periodontol 1978; 49:607-20.

7. Shearer DM, Thomson WM, Caspi A, Moffitt TE, Broadbent JM, Poulton R. Inter-generational continuity in periodontal health: findings from the Dunedin family history study. J Clin Periodontol 201 1;38:301-9.

8. O'Leary TJ, Drake RB, Crump PP, Allen MF. The incidence of recession in young males: a further study.J Periodontol 1971;42:264-7.

9. Vehkalahti M. Ocurrence of gingival recession in adults. J Periodontol 1989;60:599-603.

10. Özkavaf A, Berberoglu A, Yamalik N. An unusual cause of gingival recession: oral piercing. J Periodontol 2000; 71:1767-9.

11. Wennström JL, Lindhe J, Sinclair F, Thilander B. Some periodontal tissue reactions to orthodontic tooth movement in monkeys. J Clin Periodontol 1987;14:121-9.

12. Ruf S, Hansen K, Pancherz H. Does orthodontic proclination of lower incisors in children and adolescents cause gingival recession? American Journal of Orthodontics and Dentofacial Orthopedics 1998;114:100-6.

13. Steiner G, Pearson J, Ainamo J. Changes of the marginal periodontium as a result of labial tooth movement in momkeys. J Periodontol 1981;52:314-20.

14. Artun J, Krogstad O. Periodontal status of mandibular incisors following excessive proclination. A study in adults with surgically treated mandibular prognathism. American Journal of Orthodontic and Dentofacial Orthopedics 1987;91:225-32.

15. Chambrone L, Chambrone D, Pustiglioni FE, Chambrone LA, Lima LA. Can subepithelial connective tissue grafts be considered the gold standard procedure in the treatment of Miller Class I and II recession-type defects? J Dent 2008;36:659-71.

16. Ahathya RS, Deepalakshmi D, Ramakrishnan T, Ambalavanan N, Emmadi P. Subepithelial connective tissue grafts for the coverage of denuded root surfaces: a clinical report. Indian J Dent Res 2008;19:134-40.

17. Pagliaro U, Nieri M, Franceshi D. Evidence-based mucogingival therapy. Part 1: A critical review of the literature on root coverage procedures. J Periodontol 2003;74: $709-40$. 
18. Matas F, Sentis J, Mendieta C.Ten-year longitudinal study of gingival recession in dentists. J Clin Periodontol 2011;38:1091-8.

19. Kassab MM, Cohen RE. The etiology and prevalence of gingival recession. J Am Dent Assoc. 2003; 134:220-5.

20. Lovegrove J, Leichter J. Exposed root surface: a review of etiology, management and evidence-based outcomes of treatment. N Z Dent J 2004;100:72-81.

21. Müller HP, Eger T. Masticatory mucosa and periodontal phenotype: a review. Int J Periodontics Restorative Dent 2002;22:172-83.

22. Litonjua LA, Andreana S, Bush PJ, Cohen RE. Toothbrushing and gingival recession. Int Dent J 2003;53:67-72.

23. Susin C, Haas AN, Oppermann RV, Haugejorden O, Albandar JM. Gingival recession: epidemiology and risk indicators in a representative urban Brazilian population. J Periodontol 2004;75:1377-86.

24. Lang $N$, Löe $H$. The relationship between the width of keratinized gingiva and gingival health. J Periodontol 1972;43:623-7.
25. Kennedy J E, Bird W C, Palcanis K G, y cols. A longitudinal evaluation of varying widths of attached gingiva. J Clin Periodontol 1985;12:667-75.

26. Lekholm U, Adell R, Lindhe J, Branemark P, Ericsson B, Rockler B, et al. Marginal tissue reactions at osseointegrated titanium fixtures. A cross-sectional retrospective study. Int J Oral Maxillofac Surg 1986;15: 53-61.

27. Chen ST, Buser D. Clinical and esthetic outcomes of implants placed in postextraction sites. Int J Oral Maxillofac Implants 2009;24:186-217.

28. Chambrone L, Chambrone D, Pustiglioni FE, Chambrone LA, Lima LA. The influence of tobacco smoking on the outcomes achieved by root-coverage procedures: a systematic review. J Am Dent Assoc 2009; 140:294-306.

\section{CORRESPONDENCIA}

Antonio García Rubio

Avenida de la Estación, 23, 4 , 1

04005 Almería

Correo electrónico: antgarrub@gmail.com 